\title{
The ray transference of a reversed optical system
}

\section{WF Harris}

\author{
Department of Optometry, University of Johannesburg, PO Box 524, Auckland Park, 2006 South \\ Africa
}

<wharris@uj.ac.za>

Received 13 June 2008; revised version accepted 25 June 2008

\begin{abstract}
The optical character of an optical system is changed if the system is reversed. This is as true of eyes as it is of telescopes. In vision light traverses the eye from cornea to retina; in ophthalmoscopy the practitioner views the retina via light traversing the eye in the reverse direction. The purpose of this paper is to determine the relationship of the optical character of the reversed system to that of the system itself. The result is in terms of the ray
\end{abstract}

transference of the system. Since the ray transference gives a complete characterization of the firstorder optics of a system the analysis is complete in this sense as well. Explicit expressions are also presented for the effect of reversal on the six fundamental first-order optical properties of the system.

Keywords: reversed eye, transference, symplecticity, ray state, fundamental properties, optical system

\section{Introduction}

In vision light traverses the eye from the cornea to the retina. In ophthalmoscopy, and in many other processes of optometric and opththalmological interest, light traverses the eye in the reverse sense, from the retina and out through the cornea. Relative to the eye in vision the eye in ophthalmoscopy is a reversed optical system. It is of interest in ophthalmology and optometry, therefore, to know how the optical character of the system and the reversed system are related. Because a system's ray transference represents a complete first-order optical characterization of the system $^{1-5}$ the problem is solved completely in general if, given the transference of the system, we can obtain the transference of the reversed system. Accordingly the purpose of this paper is to derive, for an arbitrary optical system, an expression for the transference of the reversed system in terms of the transference of the system itself. Explicit expressions for the dioptric power and for all the fundamental first-order optical properties of the reversed system are also found.

Although the eye is the primary motivation for this exercise we also have in mind other systems that are reversed in optometric and ophthalmological application, telescopes and the Jackson cross-cylinder, for example.

\section{An arbitrary system}

Figure 1(a) shows an arbitrary optical system S. $\mathrm{Z}$ is a longitudinal axis: it defines the positive sense through the system. $\mathrm{T}_{0}$ is the entrance plane and $\mathrm{T}$ the exit plane of the system. The system has transference $^{3,4}$

$$
\mathbf{T}:=\left(\begin{array}{ccc}
\mathbf{A} & \mathbf{B} & \mathbf{e} \\
\mathbf{C} & \mathbf{D} & \boldsymbol{\pi} \\
\mathbf{o}^{\mathrm{T}} & \mathbf{o}^{\mathrm{T}} & 1
\end{array}\right)
$$


$\mathbf{T}$ is $5 \times 5$. Submatrices A (the dilation), B (the disjugacy), $\mathbf{C}$ (the divergence) and $\mathbf{D}$ (the divarication) are $2 \times 2$ and submatrices $\mathbf{e}$ (the transverse translation) and $\pi$ (the deflection) are $2 \times 1$. The six submatrices are the fundamental first-order optical properties of the system. $\mathbf{e}$ and $\pi$ are a consequence of prismatic and decentred elements in the system. $\mathbf{o}$ is the $2 \times 1$ null matrix and $\mathbf{o}^{\mathrm{T}}$ is its matrix transpose.

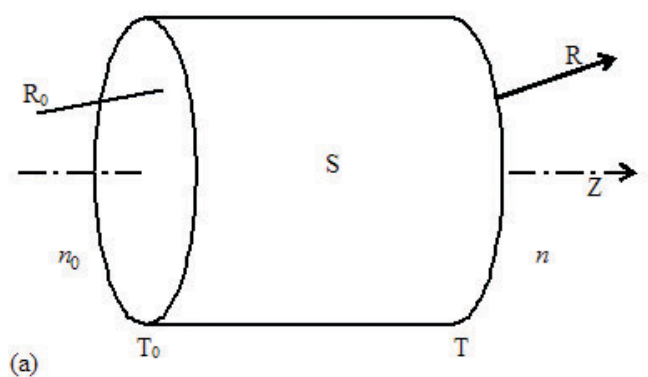

(b)

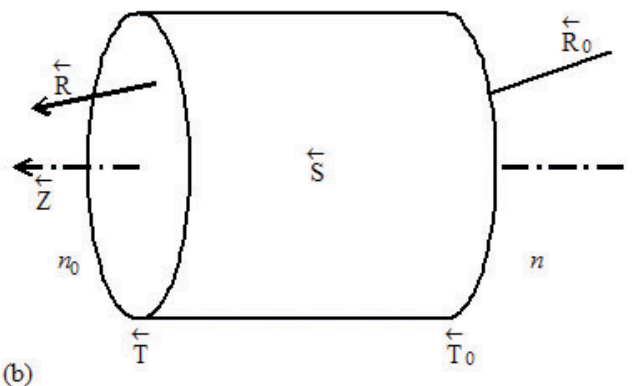

Figure 1 An arbitrary optical system $\mathrm{S}$ in (a) has longitudinal axis $\mathrm{Z}$ and entrance and exit planes $\mathrm{T}_{0}$ and $\mathrm{T}$ respectively. When applied to the eye $T_{0}$ may be immediately in front of the cornea and $\mathrm{T}$ immediately in front of the retina. The medium immediately before the system has index $n_{0}$ and the medium immediately after it has index $n$. An arbitrary ray $\mathrm{R}_{0} \mathrm{R}$ traverses the system. The ray is reversed in (b) to become ray $\mathrm{R}_{0} \mathrm{R}$. The system becomes the reversed system $\overleftarrow{\mathrm{S}}$ with longitudinal axis $\overleftarrow{Z}$ and entrance and exit planes $\overleftarrow{\mathrm{T}}_{0}$ and $\stackrel{\leftarrow}{\mathrm{T}}$.

A ray $\mathrm{R}_{0} \mathrm{R}$ traverses the system in Figure 1(a). At incidence onto $\mathrm{T}_{0}$ it has state

$$
\gamma_{0}:=\left(\begin{array}{c}
\mathbf{y}_{0} \\
\boldsymbol{\alpha}_{0} \\
1
\end{array}\right)
$$

and at emergence from $\mathrm{T}$ it has state

$$
\gamma:=\left(\begin{array}{c}
\mathbf{y} \\
\alpha \\
1
\end{array}\right)
$$

the two states being related by

$$
\mathbf{T} \boldsymbol{\gamma}_{0}=\gamma
$$

The incident and emergent states $\gamma_{0}$ and $\gamma$ are $5 \times 1$. Submatrix $\mathbf{y}_{0}$ represents the transverse position of the ray on the entrance plane $T_{0}$ relative to longitudinal axis $Z$. It is $2 \times 1$ and has the form

$\mathbf{y}_{0}:=\left(\begin{array}{l}y_{10} \\ y_{20}\end{array}\right)$

where $y_{10}$ is the horizontal coordinate and $y_{20}$ the vertical coordinate. Similarly $\mathbf{y}$ represents the position of the ray at emergence from the exit plane T. Submatrix $\boldsymbol{\alpha}_{0}$ is the reduced inclination of ray segment $\mathrm{R}_{0}$ at incidence onto $\mathrm{T}_{0}$ relative to $\mathrm{Z}$. It is related to the incident inclination $\mathbf{a}_{0}$ by

$\boldsymbol{\alpha}_{0}:=n_{0} \mathbf{a}_{0}$

where $n_{0}$ is the index of refraction immediately before the entrance plane $\mathrm{T}_{0}$. Like $\mathbf{y}_{0}$ in Equation $5 \boldsymbol{\alpha}_{0}$ and $\mathbf{a}_{0}$ have horizontal and vertical coordinates. For example $a_{10}$ is the horizontal component of the angle of incidence of ray segment $\mathrm{R}_{0}$ onto $\mathrm{T}_{0}$ and $a_{20}$ is the vertical component. Similarly submatrix $\alpha$ is the reduced inclination of segment $\mathrm{R}$ of the ray at emergence from exit plane $\mathrm{T}$ and it is related to the emergent inclination $\mathbf{a}$ by

$\alpha:=n \mathbf{a}$

where $n$ is the index immediately after exit plane T.

\section{The reversed system}

We now reverse the general direction of the light though the system (Figure 1(b)). This requires reversal of the longitudinal axis: longitudinal axis $\mathrm{Z}$ becomes longitudinal axis $\overleftarrow{Z}$. We say that system $S$ has become reversed system $\overleftarrow{S}$. The exit plane $T$ of system $\mathrm{S}$ becomes the entrance plane $\mathrm{T}$ of $\mathrm{S}$ and the entrance plane $\overleftarrow{\mathrm{T}}_{0}$ of S becomes the exit plane $\overleftarrow{\mathrm{T}}$ of $\overleftarrow{\mathrm{S}}$. Reversed system $\overleftarrow{\mathrm{S}}$ has transference

$$
\overleftarrow{\mathbf{T}}:=\left(\begin{array}{ccc}
\overleftarrow{\mathbf{A}} & \overleftarrow{\mathbf{B}} & \overleftarrow{\mathbf{e}} \\
\overleftarrow{\mathbf{C}} & \overleftarrow{\mathbf{D}} & \overleftarrow{\pi} \\
\mathbf{o}^{\mathrm{T}} & \mathbf{o}^{\mathrm{T}} & 1
\end{array}\right)
$$

We shall also refer to $\overleftarrow{\mathbf{T}}$ as the reversed transference. 
Similarly $\overleftarrow{\mathbf{A}}, \overleftarrow{\mathbf{B}}, \overleftarrow{\mathbf{C}}, \overleftarrow{\mathbf{D}}, \overleftarrow{\mathbf{e}}$ and $\overleftarrow{\boldsymbol{\pi}}$ are the fundamental properties of the reversed system; we shall also call them reversed properties.

Our goal is to determine the reversed transference $\overleftarrow{\mathbf{T}}$ in terms of the (unreversed) transference $\mathbf{T}$ and, hence, to determine the reversed fundamental properties in terms of (unreversed) fundamental properties.

Reversing ray $\mathrm{R}_{0} \mathrm{R}$ results in ray $\mathrm{R}_{0} \stackrel{\leftarrow}{\mathrm{R}}$ (Figure 1(b)). At incidence onto $\mathrm{T}_{0}$ it has state

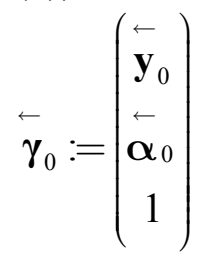

and at emergence from $\stackrel{\leftarrow}{\mathrm{T}}$ state

$$
\overleftarrow{\gamma}:=\left(\begin{array}{c}
\leftarrow \\
\mathbf{y} \\
\leftarrow \\
\alpha \\
1
\end{array}\right)
$$

the two states being related by

$$
\overleftarrow{\mathbf{T}} \boldsymbol{\gamma}_{0}=\overleftarrow{\gamma}
$$

The relation between the system and the reversed system

Consider incident ray segment $\mathrm{R}_{0}$. After reversal it becomes emergent segment $\stackrel{\leftarrow}{\mathrm{R}}$. More particularly we consider the vertical coordinates $y_{20}$ and $y_{2}$ of the position vectors $\mathbf{y}_{0}$ and $\mathbf{y}$ of the two segments. Clearly we have

$$
\overleftarrow{y}_{2}=y_{20} \text {. }
$$

On the other hand for the horizontal coordinates we find

$\overleftarrow{y}_{1}=-y_{10}$.

This follows because, for example, a ray position to the right in system $\mathrm{S}$ becomes a ray position to the left in system $\overleftarrow{\mathrm{S}}$. Combining Equations 12 and 13 we obtain

$$
\left(\begin{array}{l}
\leftarrow \\
y_{1} \\
\leftarrow \\
y_{2}
\end{array}\right)=\left(\begin{array}{c}
-y_{10} \\
y_{20}
\end{array}\right)
$$

which can be written conveniently as

$\mathbf{y}=-\mathbf{J y}_{0}$

where

$\mathbf{J}:=\left(\begin{array}{cc}1 & 0 \\ 0 & -1\end{array}\right)$.

We now consider the reduced inclinations of ray segments $\mathrm{R}_{0}$ and $\stackrel{\leftarrow}{\mathrm{R}}$. On reversal a ray inclined upward becomes a ray inclined downward and a ray inclined to the right remains inclined to the right. It follows that

$\overleftarrow{\mathbf{\alpha}}=\mathbf{J} \boldsymbol{\alpha}_{0}$.

Finally we consider emergent ray segment R. It becomes incident segment $\mathrm{R}_{0}$ on reversal. We obtain

$\overleftarrow{\mathbf{y}}_{0}=-\mathbf{J y}$.

and

$\overleftarrow{\alpha}_{0}=\mathbf{J} \boldsymbol{\alpha}$.

Substitution from Equations 18 and 19 into Equation 9 we obtain

$$
\overleftarrow{\boldsymbol{\gamma}}_{0}=\left(\begin{array}{c}
-\mathbf{J y} \\
\mathbf{J} \mathbf{\alpha} \\
1
\end{array}\right)
$$

which we can write as

$\overleftarrow{\gamma}_{0}=\mathbf{N} \boldsymbol{\gamma}$

where

$\mathbf{N}:=\left(\begin{array}{ccc}-\mathbf{J} & \mathbf{O} & \mathbf{0} \\ \mathbf{O} & \mathbf{J} & \mathbf{0} \\ \mathbf{0}^{\mathrm{T}} & \mathbf{0}^{\mathrm{T}} & 1\end{array}\right)$

is $5 \times 5$, diagonal and involutary. ( $\mathbf{M}$ is involutary if $\mathbf{M}^{2}=\mathbf{I}$ where $\mathbf{I}$ is an identity matrix. ${ }^{6}$ Such matrices are also called unipotent. ${ }^{7}$ ) $\mathbf{O}$ is the $2 \times 2$ null matrix.

Similarly, substituting from Equations 15 and 17 into Equation 10 we find that

$\boldsymbol{\gamma}=\mathbf{N} \boldsymbol{\gamma}_{0}$.

We now substitute from Equations 21 and 23 into 
Equation 11:

$\mathbf{T} \mathbf{N} \boldsymbol{\gamma}=\mathbf{N} \boldsymbol{\gamma}_{0}$.

Because $\mathbf{N}^{-1}=\mathbf{N}$ we can rewrite Equation 24 as

$\mathbf{N T} \mathbf{T} \boldsymbol{\gamma}=\gamma_{0}$.

But from Equation 4

$\mathbf{T}^{-1} \boldsymbol{\gamma}=\gamma_{0}$.

Comparing Equations 25 and 26 we see that

$\mathbf{N} \overleftarrow{\mathbf{T}} \mathbf{N}=\mathbf{T}^{-1}$

from which it follows that

$\overleftarrow{\mathbf{T}}=\mathbf{N} \mathbf{T}^{-1} \mathbf{N}$.

Equation 28 is the solution we have been seeking, namely the transference $\overleftarrow{\mathbf{T}}$ of reversed system $\overleftarrow{\mathrm{S}}$ in terms of the transference $\mathbf{T}$ of (unreversed) system $\mathbf{S}$.

Because of symplecticity ${ }^{8} \mathbf{T}^{-1}$ exists uniquely for every system. Hence, by Equation 28 we see that the reversed transference $\underset{\mathbf{T}}{\overleftarrow{ }}$ also exists uniquely for every system. Reversing a system twice returns it to the way it was which is in agreement with

$$
\stackrel{\leftarrow}{\mathbf{T}}=\mathbf{T},
$$

a result that follows from applying Equation 28 twice.

The relation between the fundamental properties of the system and the reversed system

Using the fact that the block $\left(\begin{array}{ll}\mathbf{A} & \mathbf{B} \\ \mathbf{C} & \mathbf{D}\end{array}\right)$ is symple-

ctic one can readily show by direct multiplication that

$\mathbf{T}^{-1}=\left(\begin{array}{ccc}\mathbf{D}^{\mathrm{T}} & -\mathbf{B}^{\mathrm{T}} & -\mathbf{D}^{\mathrm{T}} \mathbf{e}+\mathbf{B}^{\mathrm{T}} \boldsymbol{\pi} \\ -\mathbf{C}^{\mathrm{T}} & \mathbf{A}^{\mathrm{T}} & \mathbf{C}^{\mathrm{T}} \mathbf{e}-\mathbf{A}^{\mathrm{T}} \boldsymbol{\pi} \\ \mathbf{o}^{\mathrm{T}} & \mathbf{o}^{\mathrm{T}} & 1\end{array}\right)$.

Substituting into Equation 28 one finds that

$\overleftarrow{\mathbf{T}}=\left(\begin{array}{ccc}\mathbf{J D}^{\mathrm{T}} \mathbf{J} & \mathbf{J B}^{\mathrm{T}} \mathbf{J} & \mathbf{J D}^{\mathrm{T}} \mathbf{e}-\mathbf{J} \mathbf{B}^{\mathrm{T}} \boldsymbol{\pi} \\ \mathbf{J C}^{\mathrm{T}} \mathbf{J} & \mathbf{J A}^{\mathrm{T}} \mathbf{J} & \mathbf{J C}^{\mathrm{T}} \mathbf{e}-\mathbf{J} \mathbf{A}^{\mathrm{T}} \boldsymbol{\pi} \\ \mathbf{o}^{\mathrm{T}} & \mathbf{o}^{\mathrm{T}} & 1\end{array}\right)$.

Hence the fundamental properties of the reversed system are

$$
\begin{aligned}
& \overleftarrow{\mathbf{A}}=\mathbf{J} \mathbf{D}^{\mathrm{T}} \mathbf{J} \\
& \overleftarrow{\mathbf{B}}=\mathbf{J} \mathbf{B}^{\mathrm{T}} \mathbf{J} \\
& \overleftarrow{\mathbf{C}}=\mathbf{J} \mathbf{C}^{\mathrm{T}} \mathbf{J} \\
& \overleftarrow{\mathbf{D}}=\mathbf{J} \mathbf{A}^{\mathrm{T}} \mathbf{J} \\
& \overleftarrow{\mathbf{e}}=\mathbf{J} \mathbf{D}^{\mathrm{T}} \mathbf{e}-\mathbf{J} \mathbf{B}^{\mathrm{T}} \boldsymbol{\pi} \\
& \overleftarrow{\boldsymbol{\pi}}=\mathbf{J} \mathbf{C}^{\mathrm{T}} \mathbf{e}-\mathbf{J} \mathbf{A}^{\mathrm{T}} \boldsymbol{\pi} .
\end{aligned}
$$

By definition the dioptric power matrix is ${ }^{9}$

$\mathbf{F}:=-\mathbf{C}$.

Hence, from Equation 34, we have

$$
\overleftarrow{\mathbf{F}}=\mathbf{J F}^{\mathrm{T}} \mathbf{J}
$$

If we write disjugacy $\mathbf{B}$ in terms of its elements as $\mathbf{B}=\left(\begin{array}{ll}b_{11} & b_{12} \\ b_{21} & b_{22}\end{array}\right)$

and evaluate the right-hand side of Equation 33 we find that

$\overleftarrow{\mathbf{B}}=\left(\begin{array}{cc}b_{11} & -b_{21} \\ -b_{12} & b_{22}\end{array}\right)$.

In other words, reversing the system interchanges the two off-diagonal elements of $\mathbf{B}$ and changes their signs. The diagonal elements of $\mathbf{B}$ are unchanged. The same applies in the case of divergence $\mathbf{C}$ as Equation 34 shows and power $\mathbf{F}$ as Equation 39 shows. The same also applies in the case of dilation $\mathbf{A}$ and divarication $\mathbf{D}$ except that $\mathbf{A}$ and $\mathbf{D}$ are also interchanged (Equations 32 and 35). Equations 36 and 37 show that the effect of reversal on the transverse translation e and deflection $\pi$ is more complicated.

If we expand disjugacy as

$\mathbf{B}=B_{\mathrm{I}} \mathbf{I}+B_{\mathrm{J}} \mathbf{J}+B_{\mathrm{K}} \mathbf{K}+B_{\mathrm{L}} \mathbf{L}$,

where

$\mathbf{K}:=\left(\begin{array}{ll}0 & 1 \\ 1 & 0\end{array}\right)$

and

$\mathbf{L}:=\left(\begin{array}{cc}0 & 1 \\ -1 & 0\end{array}\right)$

we see from Equation 33 that

$\overleftarrow{\mathbf{B}}=B_{\mathrm{I}} \mathbf{I}+B_{\mathrm{J}} \mathbf{J}-B_{\mathrm{K}} \mathbf{K}+B_{\mathrm{L}} \mathbf{L}$ 
In other words the only effect reversal has on disjugacy is to reverse the sign of the coefficient of $\mathbf{K}$, that is, to reverse the sign of the oblique antistigmatic component. The same applies to divergence $\mathbf{C}$ and power F. Again it also applies to $\mathbf{A}$ and $\mathbf{D}$ except that $\mathbf{A}$ and D are also interchanged.

Systems with $\mathbf{A}=\mathbf{O}$ cause parallel incident rays to focus in the exit plane of the system; they are exitplane focal systems. If the system is an eye then it is emmetropic. ${ }^{5}$ Systems with $\mathbf{D}=\mathbf{O}$ are entranceplane focal. Equation 35 shows that, as expected, an exit-plane focal system become entrance-plane focal when reversed and Equation 32 that the reverse is also true. A conjugate system ( $\mathbf{B}=\mathbf{O}$ ) remains a conjugate system on reversal in agreement with Equation 33 and an afocal system ( $\mathbf{C}=\mathbf{O}$ ) remains afocal in agreement with Equation 34. Equations 36 and 37 show that a centred system $(\mathbf{e}=\mathbf{0}$ and $\boldsymbol{\pi}=\mathbf{0})$ remains centred. However it follows from Equation 36 that a system with $\mathbf{e}=\mathbf{0}$ does not in general imply $\overleftarrow{\mathbf{e}}=\mathbf{0}$. Similarly (Equation 37 ) a system with $\boldsymbol{\pi}=\mathbf{0}$ does not necessarily imply $\overleftarrow{\boldsymbol{\pi}}=\mathbf{0}$, a case that is illustrated in the following example. Consider a particular decentred thin lens followed by a homogeneous gap and a thin prism. The transference is

$$
\mathbf{T}=\left(\begin{array}{ccc}
\mathbf{I} & \mathbf{O} & \mathbf{0} \\
\mathbf{O} & \mathbf{I} & -\boldsymbol{\pi} \\
\mathbf{0}^{\mathrm{T}} & \mathbf{0}^{\mathrm{T}} & 1
\end{array}\right)\left(\begin{array}{ccc}
\mathbf{I} & \zeta \mathbf{I} & \mathbf{0} \\
\mathbf{O} & \mathbf{I} & \mathbf{0} \\
\mathbf{o}^{\mathrm{T}} & \mathbf{o}^{\mathrm{T}} & 1
\end{array}\right)\left(\begin{array}{ccc}
\mathbf{I} & \mathbf{O} & \mathbf{0} \\
\mathbf{C} & \mathbf{I} & \boldsymbol{\pi} \\
\mathbf{0}^{\mathrm{T}} & \mathbf{o}^{\mathrm{T}} & 1
\end{array}\right)
$$

or

$$
\mathbf{T}=\left(\begin{array}{ccc}
\mathbf{I}+\zeta \mathbf{C} & \zeta \mathbf{I} & \zeta \boldsymbol{\pi} \\
\mathbf{C} & \mathbf{I} & \mathbf{0} \\
\mathbf{0}^{\mathrm{T}} & \mathbf{0}^{\mathrm{T}} & 1
\end{array}\right)
$$

where the divergence $\mathbf{C}$ is symmetric and $\mathbf{I}$ is the $2 \times 2$ identity matrix. It follows from Equations 32 to 37 that the reversed transference is

$$
\overleftarrow{\mathbf{T}}=\left(\begin{array}{ccc}
\mathbf{I} & \zeta \mathbf{I} & \zeta \mathbf{J} \boldsymbol{\pi} \\
\mathbf{J C J} & \mathbf{I}+\zeta \mathbf{J C} \mathbf{J} & \zeta \mathbf{J C} \boldsymbol{\pi} \\
\mathbf{o}^{\mathrm{T}} & \mathbf{0}^{\mathrm{T}} & 1
\end{array}\right) .
$$

Thus, while the system has a null deflection (Equation 47), the reversed system does not (Equation 48). For a thin system

$$
\overleftarrow{\mathbf{T}}=\left(\begin{array}{ccc}
\mathbf{I} & \mathbf{O} & \mathbf{0} \\
\mathbf{J C J} & \mathbf{I} & -\mathbf{J} \boldsymbol{\pi} \\
\mathbf{o}^{\mathrm{T}} & \mathbf{0}^{\mathrm{T}} & 1
\end{array}\right)
$$

The effect of the operation $\mathbf{J C J}$ on $\mathbf{C}$ in Equations 48 and 49 is merely to change the sign of the off-diagonal elements. Because of Equation 39 the same is true of the dioptric power $\mathbf{F}$.

If none of the elements of the system is astigmatic then the four $2 \times 2$ fundamental properties are scalar matrices and Equation 31 reduces to

$\overleftarrow{\mathbf{T}}=\left(\begin{array}{ccc}D \mathbf{I} & B \mathbf{I} & D \mathbf{J e}-B \mathbf{J} \boldsymbol{\pi} \\ C \mathbf{I} & A \mathbf{I} & C \mathbf{J} \mathbf{e}-A \mathbf{J} \boldsymbol{\pi} \\ \mathbf{o}^{\mathrm{T}} & \mathbf{0}^{\mathrm{T}} & 1\end{array}\right)$.

Thus the disjugacy, the divergence and the power are unchanged by reversal while the dilation becomes the divarication and vice versa.

In Gaussian optics the transference reduces to a $3 \times 3$ matrix. The reversed transference can be obtained from the analysis above by ignoring the horizontal coordinate. The result turns out to be

$$
\overleftarrow{\mathbf{T}}=\left(\begin{array}{ccc}
D & B & -D e+B \pi \\
C & A & -C e+A \pi \\
0 & 0 & 1
\end{array}\right) .
$$

\section{Conclusion}

Equation 28 answers the problem posed in this paper. It shows explicitly what happens to the transference of an eye, or any other optical system, when the system is reversed. $\mathbf{N}$ in that equation is the diagonal matrix defined by Equation 22. The six fundamental properties of the system are changed according to Equations 32 to 37 where $\mathbf{J}$ is the matrix defined by Equation 16. When astigmatism is ignored the reversed transference reduces to that given by Equation 50 and, in Gaussian optics, by Equation 51. In the absence of astigmatism disjugacy, divergence and power are unchanged by reversal (Equations 50 and 51). That is not true in the presence of astigmatism although, in the case of these properties, the change is only that the oblique antistigmatic component has its sign changed as illustrated by Equation 45 .

\section{Acknowledgements}

I thank SD Mathebula for commenting on the manuscript. 


\section{References}

1. Keating MP. A system matrix for astigmatic optical systems: I. Introduction and dioptric power relations. $\mathrm{Am} \mathrm{J}$ Optom Physiol Opt 198158 810-9.

2. Keating MP. A system matrix for astigmatic optical systems: II. Corrected systems including an astigmatic eye. Am J Optom Physiol Opt 198158 919-29.

3. Harris WF. Paraxial ray tracing through noncoaxial astigmatic optical systems, and a $5 \times 5$ augmented system matrix. Optom Vis Sci 199471 282-5.

4. Harris WF. Magnification, blur, and ray state at the retina for the general eye with and without a general optical instrument in front of it. 1. Distant objects. Optom Vis Sci 200178 888-900.

5. Harris WF. A unified paraxial approach to astigmatic optics. Optom Vis Sci 199976 480-499.

6. Bernstein DS. Matrix Mathematics. Princeton University Press, Princeton, 2005, 81.

7. Lütkepohl H. Handbook of Matrices. Wiley, Chichester, 1996, 254.

8. Kauderer M. Symplectic Matrices: First Order Systems and Special Relativity. World Scientific, Singapore, 1994, 9.

9. Harris WF. Dioptric power: its nature and its representation in three- and four-dimensional space. Optom Vis Sci 1997 74 349-366. 